\title{
Oxygen therapy practices in the acutely ill medical patients: A social media-based nationwide study of clinicians' preferences and summary of current recommendations
}

\author{
George Karlis, ${ }^{1}$ Dimitrios Barouxis, ${ }^{2}$ Georgios Georgiopoulos, ${ }^{3}$ Panagiota Mitropoulou, ${ }^{4}$ \\ Zafeiria Mastora, ${ }^{5}$ Theodoros Xanthos ${ }^{6}$
}

${ }^{1}$ Intensive Care Unit, General Hospital of Rhodes "Andreas Papandreou"; ${ }^{2}$ Cardiology Department, Laiko General Hospital, Athens; ${ }^{3}$ Department of Clinical Therapeutics, National and Kapodistrian University of Athens, Greece; ${ }^{4}$ Queen Alexandra Hospital, Portsmouth, United Kingdom; ${ }^{5} 1^{\text {st }}$ Department of Intensive Care Medicine, National and Kapodistrian University of Athens, Greece; ${ }^{6}$ European University Cyprus, School of Medicine, Nicosia, Cyprus

\begin{abstract}
Oxygen is the most commonly used drug in emergency medicine. The aim of this study was to identify healthcare professionals' preferences regarding oxygen therapy in common medical emergencies. An online 9-part-questionnaire was distributed through Facebook to doctors and nurses working in Greek hospitals. The questionnaire included background information of the respondents and addressed individual preferences regarding best oxygenation parameter and oxygen targets in specific acute settings. We received 678 responses and we included 663 in our analysis. We found significant differences between doctors' and nurses' attitudes towards oxygenation targets in ARDS, sepsis, acute coronary syndrome, and post cardiac-arrest patients. Nurses preferred a more conservative oxygen strategy compared to doctors. Furthermore, nurses favor $\mathrm{SaO}_{2}$ as the best oxygenation parameter, while doctors prefer $\mathrm{PaO}_{2}$. In our survey, the type of
\end{abstract}

\footnotetext{
Correspondence: George Karlis, Attavyrou 24, 85100, Rodos, Greece E-mail: georgekarlis@yahoo.com

Tel: +306937582535

Key words: Oxygen therapy; Emergency medicine; Survey

Contributions: GK, idea conception and manuscript writing; DB, data collection and reference search; GG, statistical analysis and manuscript writing; PM, reference search and data collection; ZM, manuscript reviewing; TX, manuscript reviewing.

Conflict of interest: The authors declare no potential conflict of interests.

Ethics approval and consent to participate: The manuscript does not contain any individual person's data in any form.

Dedication: The article is dedicated to Mike Karlis Jr.

Received for publication: 2 February 2020

Accepted for publication: 18 May 2020.

This work is licensed under a Creative Commons Attribution 4.0 License (by-nc 4.0).

CCopyright: the Author(s), 2020

Licensee PAGEPress, Italy

Emergency Care Journal 2020; 16:8867

doi:10.4081/ecj.2020.8867
}

hospital and department of the respondents did not affect the preferred oxygen strategy. Social media-based survey research is feasible and effective. In this single country study, doctors showed a tendency to liberally administer oxygen in acutely ill medical patients. On the other hand, Greek nurses preferred a more conservative approach.

\section{Introduction}

Oxygen therapy represents a central focus of emergency care. Nevertheless, the debate among healthcare professionals about the optimal targets of oxygenation is constant. Supplemental oxygen is universally administered to acutely ill patients to treat life-threatening hypoxia, but it is now well documented that it may impose potential harmful effects. These include direct cellular damage by reactive oxygen species, pulmonary absorption atelectasis, lung inflammation/injury, and hyperoxemic vasoconstriction. ${ }^{1,2}$ Sometimes the benefits of attempting to fully reverse arterial hypoxemia may be outweighed by the risks associated with high oxygen concentrations. ${ }^{3}$ However, it is not known whether the primary culprit is the fraction of inspired oxygen $\left(\mathrm{FiO}_{2}\right)$ itself or the systemic effects of hyperoxemia.

Although recommendations for oxygen therapy in the adult acutely ill patients do exist, the optimal disease specific targets have not been established. Arterial blood gases (ABGs) and partial pressure of oxygen $\left(\mathrm{PaO}_{2}\right)$ have been historically considered the gold standard for assessment of oxygenation. Measurement of $\mathrm{PaO}_{2}$ though, requires an arterial puncture, can be challenging in some patients and it is not ideal for monitoring. In the new era, oxyhemoglobin saturation measured by pulse oximetry $\left(\mathrm{SpO}_{2}\right.$ or $\mathrm{SaO}_{2}$ ) has become a useful adjunct for assessment of oxygen status and it is uniformly used for non-invasive monitoring at the bedside. $^{4}$

Currently, the liberal versus conservative approach in oxygen therapy has been a matter of controversy. Data from randomized controlled trials (RCTs) have been inconclusive. A recent metaanalysis of 25 RCTs concluded that liberal oxygen therapy increased mortality in acutely ill adults. ${ }^{5}$ However, these results were disputed on the basis of poor fragility index of the included studies. ${ }^{6}$ In this setting, clinicians' preferences and actual clinical practice regarding oxygen therapy are of significant interest.

We conducted a national survey seeking to explore the attitudes and practices of doctors and nurses working in Greek hospitals. The goal of the study was to quantify current practice by clar- 
ifying opinions on $\mathrm{PaO}_{2}$ versus $\mathrm{SaO}_{2}$ as a preferred oxygenation parameter and preferences of oxygen therapy targets in specific acute clinical scenarios. We also reviewed the current international guidelines about oxygen treatment in these scenarios.

\section{Material and Methods}

We produced a 9-part-questionnaire in Greek regarding oxygen administration in acutely ill adults. The questionnaire consisted of two sections. The first section (Q1 to Q3) included background information of the respondents, namely role (specialized doctor, resident doctor, nurse), department (internal medicine section, surgical section/operating room, intensive care unit/coronary care unit, emergency department), and type of hospital (university, public, private). The second section (Q4 to Q9) addressed individual preferences regarding best oxygenation parameter $\left(\mathrm{PaO}_{2}\right.$ or $\mathrm{SaO}_{2}$ ) and oxygen targets in terms of $\mathrm{SaO}_{2}$ range in specific acute settings, namely COPD exacerbation, ARDS, sepsis, acute coronary syndrome, and post-cardiac arrest.

The questionnaire was pilot-tested in ten healthcare professionals (five doctors and five nurses) to ensure understanding and correct interpretation (face validity). Two questions needed revision and were then approved by all the pilot participants.

From 29 June to 20 July 2019 the web-based questionnaire (SurveyMonkey ${ }^{\circledR}$, Dublin, Ireland) was distributed through Facebook social media platform. The link to the survey was posted to the two largest Facebook groups for doctors and nurses working in Greece. The title of the questionnaire was "Oxygen therapy targets in the emergency department, the intensive care unit and the ward". A brief description was posted along with the link: "The target population of the survey is doctors and nurses working in Greek hospitals and it is intended to assess individual preferences and practices regarding oxygen therapy and not knowledge of guidelines. This is an anonymous questionnaire and average completion time is 1 minute". A reminder post was uploaded to each group 10 days after the original post. The survey was closed on 20 July 2019

No specific ethical permissions were required for this survey. Participation was voluntary and completion was regarded as informed consent. All collected data were anonymous. The IP address of the respondents was processed by the website (SurveyMonkey®) to exclude duplicate answers. We did not have any access to the IP addresses of the respondents.

Results were analyzed using descriptive statistics. Nominal/ordinal variables are expressed in absolute and percentage value (\%). Differences in oxygenation target (i.e. 88-92 vs $92-$ 96 vs $96-100 \%$ ) overall and in terms of specific clinical situations among subgroups of interest, including role, department, and type of hospital, were assessed by the non-parametric chi-squared test. Results are provided as percentages per row and column and illustrated with bar charts. Post hoc analysis indicated that our sample size had adequate power (more than $80 \%$ ) to detect differences equal or more than $10 \%$ in percentage scale, corresponding to a small to moderate effect size equal to 0.2 according to Cohen's conventions, ${ }^{7}$ in target oxygenation preference among different groups. Power considerations were performed with GPower 3.1.9.4. ${ }^{8}$ Statistical analysis was conducted with STATA package, version 11.1 (StataCorp, College Station, Texas USA). The level of statistical significance was set at $\mathrm{p}<0.05$.

\section{Results}

We received 678 responses. Fifteen of them were not full (respondents skipped one or more questions) and were excluded from the study. We analyzed the responses of 663 healthcare professionals. Of them, 392 were nurses $(59.13 \%)$ and 271 doctors $(40.87 \%)$. Doctors were further divided in specialized doctors $(149,22.47 \%)$ and residents or doctors in training $(122,18.40 \%)$. The majority of the respondents $(38.01 \%)$ work in internal medicine departments and in public hospitals $(58.82 \%)$. The summary of all survey data is depicted in Figure 1.

The preferred oxygenation parameter is $\mathrm{PaO}_{2}$ for $55.05 \%$ of the respondents and $\mathrm{SaO}_{2}$ for $44.95 \%$. However, in the subgroup of nurses $42.86 \%$ prefer $\mathrm{PaO}_{2}$ whereas $57.14 \%$ prefer $\mathrm{SaO}_{2}$. This difference was statistically significant $(p<0.001)$. Between group comparisons of the respondents' background with respect to the preferred $\mathrm{SaO}_{2}$ target in specific acute settings (COPD exacerbation, ARDS, sepsis, acute coronary syndrome, and post-cardiac arrest) were made. There were no differences in the answers between type of hospital subgroups (university, public, private). The department (internal medicine section, surgical section/operating room, intensive care unit/coronary care unit, emergency department) did not affect significantly the preferred oxygenation targets, with the exception of ARDS. Indeed, respondents working in the intensive care unit/coronary care unit and the emergency
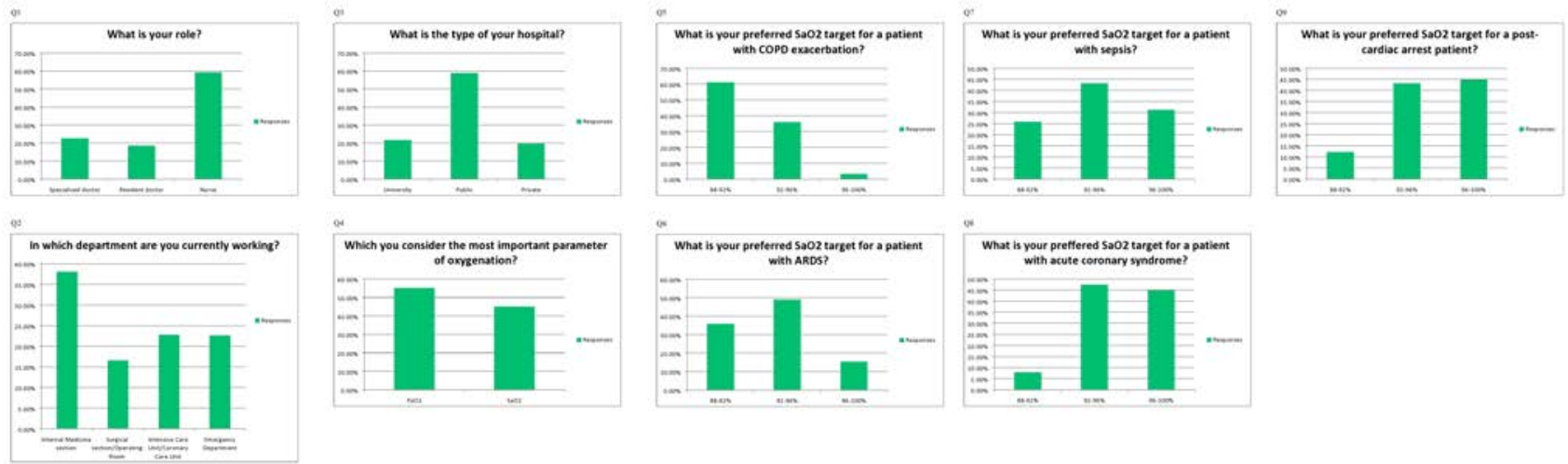

Figure 1. Summary of all survey data. 
department preferred a more conservative approach $(\mathrm{p}=0.041)$ in the ARDS patient. Interestingly, when the analysis took into consideration the role of the respondents (specialized doctor, resident doctor, nurse) the preferred oxygenation targets differed significantly in all clinical scenarios. Nurses' responses showed a preference for lower $\mathrm{SaO}_{2}$ targets overall compared to doctors. In Figure 2 we present the comparisons by role.

\section{Current recommendations regarding oxygen therapy}

Oxygen is the most commonly used drug in acute medicine. Yet prior to 2008, there was no formal guidance available for the safe use of oxygen. Ambulance staff and emergency department physicians often failed to optimize oxygen therapy, rather by using a "one size fits all" approach.

According to British Thoracic Society (BTS) guidelines for emergency oxygen use in adult patients, a target saturation of 94$98 \%$ is recommended for most acutely ill patients and $88-92 \%$ for those at risk of hypercapnic respiratory failure. ${ }^{10}$ In Table 1 we summarize the latest international recommendations for oxygen therapy targets in common medical emergencies such as COPD exacerbation, ARDS, acute coronary syndromes, stroke, sepsis/septic shock, post-cardiac arrest. ${ }^{11-15}$

\section{Discussion}

This nationwide survey showed that nurses prefer $\mathrm{SaO}_{2}$ as the target oxygenation parameter, while doctors prefer $\mathrm{PaO}_{2}$. In addition, our survey demonstrated that nurses target lower oxygen saturations in acute illness, thus showing a preference for a more conservative oxygen therapy approach compared to doctors. For instance, in ARDS and sepsis, only $9.44 \%$ and $23.98 \%$ of nurses respectively, would target an $\mathrm{SaO}_{2} \geq 96 \%$.

In the majority of hospital settings worldwide, nurses frequently manage oxygen therapy but they report lack of initiative to routinely escalate it without a doctor's request or in the absence of specific medical orders. ${ }^{16}$ It seems that nurses in some settings are less likely to independently titrate oxygen to their own $\mathrm{SaO}_{2}$ target, but more likely to independently treat a falling $\mathrm{SaO}_{2} \cdot{ }^{17}$ Furthermore there are practical issues and difficulties with changing long established behaviors in a given healthcare system. For instance, in the Greek interprofessional culture the role of the physician is more dominant in the decision-making process of patient's care. ${ }^{18}$ These might be some possible explanations for our findings in the group of nurses. However, in a corresponding study, the majority of nurses (76\%) at a district UK hospital "always" felt comfortable administering oxygen therapy while only $33 \%$ of them were aware of the consequences for patients if target $\mathrm{SaO}_{2}$ was above the prescribed limit. ${ }^{19}$ It is therefore reasonable to suggest that the setting, local/cultural aspects and the level of training
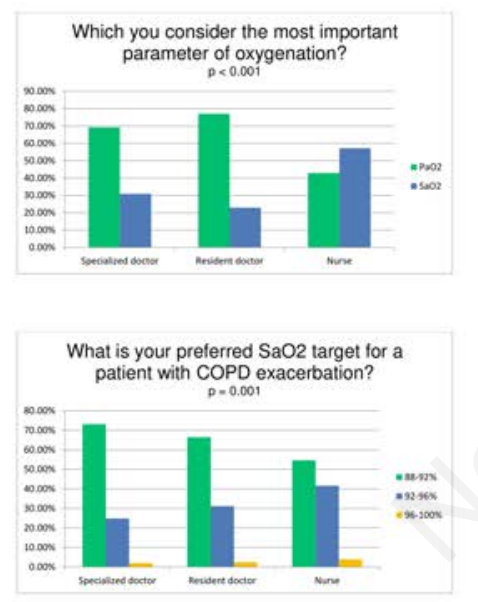

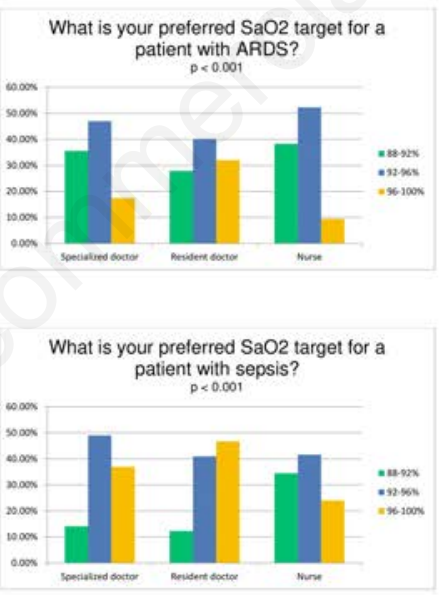

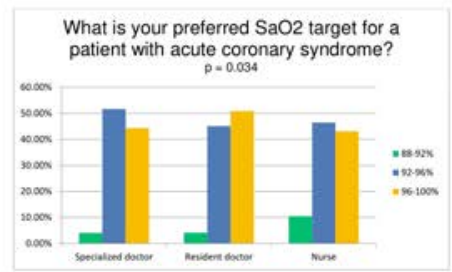

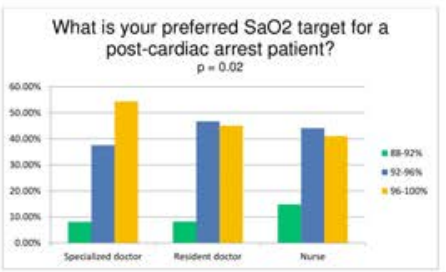

Figure 2. Comparison by role.

Table 1. Emergency oxygen use guidelines in adults.

\begin{tabular}{|c|c|c|c|c|}
\hline & Recommendation & Comments & Society & Level of evidence \\
\hline COPD exacerbation & $88-92 \%$ & Pending blood gas results & BTS & Strong \\
\hline ARDS & $\geq 88 \%$ & Evidence of harm for targets $97-100 \%$ & ATS/ESICM/SCCM & Conditional \\
\hline Acute coronary syndromes & $\geq 90 \%$ & Hyperoxia might increase myocardial injury & ESC/AHA & Low \\
\hline Stroke & $\geq 95 \%$ & Hypoxia should be avoided because it may amplify ischemic brain damage & ESO/EAN & Low \\
\hline Sepsis/septic shock & $94-98 \%$ & Oxygen consumption is impaired in the face of adequate oxygen delivery & BTS & Low \\
\hline Post-cardiac arrest & $94-98 \%$ & Hypoxia is associated with worse outcomes & ILCOR & Very low \\
\hline
\end{tabular}

COPD: Chronic Obstructive Pulmonary Disease; ARDS: Acute Respiratory Distress Syndrome; BTS: British Thoracic Society; ATS: American Thoracic Society; ESICM: European Society of Intensive Care Medicine; SCCM: Society of Critical Care Medicine; ESC: European Society of Cardiology; AHA: American Heart Association; ESO: European Stroke Organization; EAN: European Academy of Neurology; ILCOR: International Liaison Committee on Resuscitation. 
of healthcare professionals can affect their oxygen preferences and practices.

Interestingly, the vast majority of doctors $(74.9 \%)$ value $\mathrm{PaO}_{2}$ as the most important parameter of oxygenation and therefore, from the physicians' point of view, the validity of $\mathrm{SaO}_{2}$ in the emergency setting is being challenged. As previously reported, arterial blood gas confirmation of $\mathrm{SaO}_{2}$ is considered the standard of care by ICU doctors. ${ }^{20}$ Physicians tend to be more aware of the several limitations of pulse oximetry, e.g. in low perfusion states. Junior doctors consider frequent reassessment of arterial blood gases an essential part of oxygen therapy monitoring in acute patients. ${ }^{21,22}$ Moreover, in our study the group of resident doctors demonstrated the highest preference for $\mathrm{PaO}_{2}$. We compared $\mathrm{PaO}_{2}$ versus $\mathrm{SaO}_{2}$ as the preferred parameter in the evaluation of oxygenation among healthcare professionals from different departments. We did not assess the ratio of arterial oxygen partial pressure to the fraction of inspired oxygen (PF ratio) as a parameter of oxygenation. The PF ratio represents a reliable clinical indicator of hypoxaemia in the ICU setting, mainly used in the evaluation of ARDS severity as part of the Berlin definition..$^{23}$ Our survey was designed to involve healthcare professionals of different backgrounds and level of training (e.g. junior doctors). Eventually, the majority of respondents $(77.22 \%)$ reported to work outside the ICU, and therefore PF ratio might not be relevant to their everyday practice.

In our study, it was apparent that healthcare professionals in Greece are now well aware of the potential harm from targeting inappropriately high oxygen saturations in patients with an acute exacerbation of COPD. ${ }^{24}$ Indeed, $60.94 \%$ of the respondents favored an $\mathrm{SaO}_{2}$ of $88-92 \%$ and only $3.17 \%$ preferred a target $\geq 96 \%$. On the other hand, we found that acute coronary syndromes trigger a response for high oxygen saturations. The $44.80 \%$ of respondents preferred an $\mathrm{SaO}_{2}$ target of $96-100 \%$. This was even more prominent in the group of doctors, despite the fact that recent guidelines raise concerns about association of hyperoxia and increased myocardial injury. ${ }^{13}$ Classic medical teaching used to prefer the mnemonic MONA (morphine, oxygen, nitrates, aspirin) as the mainstay of acute coronary syndrome management in the emergency setting. Nowadays, there are concerns that this approach might be obsolete and inappropriate. ${ }^{25}$ A similar approach, warranting high $\mathrm{SaO}_{2}$ targets, was also evident in the current survey for patients in the early period post-cardiac arrest.

Looking at the guidelines regarding oxygen therapy in the acutely ill, the quality of evidence is low and recommendations are mainly based on observational data and consensus of experts, with the exception of patients at risk of hypercapnic respiratory failure (e.g. COPD). ${ }^{26} \mathrm{~A}$ recent meta-analysis of $25 \mathrm{RCTs}$ concluded that liberal oxygen therapy in sepsis, critical illness, stroke, trauma, myocardial infarction and cardiac arrest, defined as $\mathrm{SaO}_{2}$ range 94$99 \%$, increased in-hospital mortality without improving other patient-important outcomes. ${ }^{5}$ Moreover, conservative oxygenation strategy may also be appropriate and feasible in the group of mechanically ventilated ICU patients. ${ }^{27}$ Despite the emerging evidence favoring conservative oxygen therapy, there is a longstanding cultural norm among healthcare professionals to promptly provide supplemental oxygen to acutely ill patients, regardless of oxygen saturation values at presentation. Our study reflects this kind of liberal oxygen mentality especially among Greek physicians and calls for a change in current practice.

The widespread use and popularity of social media platforms such as Facebook, Twitter, LinkedIn among healthcare professionals offers a great opportunity for online survey research. As previously reported, these free and widely accessible websites can be used to target and recruit specific patient or healthcare professional populations. ${ }^{28,29}$ In the present online survey we managed to rapidly engage our target participants and efficiently collect data with minimal resources. Interestingly, nurses were more eager to participate in our survey compared to doctors, $59.13 \%$ versus $40.87 \%$ of respondents respectively.

The main limitation of this study is its local character, as it involved a large sample of doctors and nurses from a single European country. Furthermore, the population of the study was somewhat heterogeneous. The respondents came from different type of hospitals and different departments. In addition, the years of clinical practice of each participant were not taken into account.

In summary, we detected significant differences in oxygen therapy practices between doctors and nurses working in the Greek healthcare system. In the acutely ill medical patient, nurses showed preference for a more conservative oxygen strategy compared to doctors. Moreover, nurses considered $\mathrm{SaO}_{2}$ the most important parameter of oxygenation in contrast to doctors who preferred $\mathrm{PaO}_{2}$. To the best of our knowledge this is the first study to compare doctors' and nurses' attitudes towards oxygen therapy in common medical emergencies.

\section{Conclusions}

We report a national survey on healthcare professionals' selfreported preferences for targeting oxygenation in acute illness. Doctors overall favored higher $\mathrm{SaO}_{2}$ targets, thus a more liberal approach, despite the accumulating evidence of harm in the literature. This might have implications in clinical training and future practice. On the other hand, nurses have chosen a more conservative approach towards oxygen titration. Whether this is the effect of education and good clinical practice or a mere reflection of indecision in the absence of a specific medical order, remains unclear and warrants further evaluation. The current study confirmed again the feasibility and reliability of online survey research through social media platforms. Given the local distribution of the survey, our results need to be investigated in other healthcare systems.

\section{References}

1. Chow CW, Herrera Abreu MT, Suzuki T, et al. Oxidative stress and acute lung injury. Am J Respir Cell Mol Biol 2003;29:42731.

2. Asfar P, Singer M, Radermacher P. Understanding the benefits and harms of oxygen therapy. Intensive Care Med 2015;41:1118-21.

3. Martin DS, Grocott MP. Oxygen therapy in critical illness: precise control of arterial oxygenation and permissive hypoxemia. Crit Care Med 2013;41:423-32.

4. Pierson DJ. Oxygen in respiratory care: A personal perspective from 40 years in the field. Respir Care 2013;58:196-204.

5. Chu DK, Kim LH, Young PH, et al. Mortality and morbidity in acutely ill adults treated with liberal versus conservative oxygen therapy (IOTA): a systematic review and meta-analysis. Lancet 2018;391:1693-705.

6. Vargas M, Servillo G. Liberal versus conservative oxygen therapy in critically ill patients: using the fragility index to determine robust results. Crit Care 2019;23:132.

7. Grice JW, Barrett PT. A note on Cohen's overlapping proportions of normal distributions. Psychol Rep. 2014;115:741-7. 
8. Faul F, Erdfelder E, Lang AG, Buchner A. G*Power 3: a flexible statistical power analysis program for the social, behavioral, and biomedical sciences. Behav Res Methods 2007;39:175-91.

9. Hale KE, Gavin C, O'Driscoll BR. Audit of oxygen use in emergency ambulances and in a hospital emergency department. Emerg Med J 2008;25:773-6.

10. O'Driscoll BR, Howard LS, Davison AG. BTS guidelines for emergency oxygen use in adult patients. Thorax 2008;63:1-68.

11. O'Driscoll BR, Howard LS, Earis J, et al. BTS guidelines for oxygen use in adults in healthcare and emergency settings. Thorax 2017;72:1-90.

12. Fan E, Del Sorbo L, Goligher EC, et al. An official American Thoracic Society/European Society of Intensive Care Medicine/Society of Critical Care Medicine clinical practice guidelines: Mechanical ventilation in adult patients with acute respiratory distress syndrome. Am J Respir Crit Care Med 2017;195:1253-63.

13. Ibanez B, James S, Agewall S, et al. 2017 ESC guidelines for the management of acute myocardial infarction in patients presenting with ST-segment elevation: The task force for the management of acute myocardial infarction in patients presenting with ST-segment elevation of the European Society of Cardiology (ESC). Eur Heart J 2018;39:119-77.

14. Kobayashi A, Czlonkowska A, Ford GA, et al. European Academy of Neurology and European Stroke Organization consensus statement and practical guidance for pre-hospital management of stroke. Eur J Neurol 2018; 25:425-33.

15. Soar J, Callaway CW, Aibiki M, et al. Part 4: Advanced Life Support. 2015 International Consensus on Cardiopulmonary Resuscitation and Emergency Cardiovascular Care Science with treatment recommendations. Resuscitation 2015;95:71120.

16. Helmerhorst HJ, Schultz MJ, van der Voort PH, et al. Selfreported attitudes versus actual practice of oxygen therapy by ICU physicians and nurses. Ann Intensive Care 2014;4:23.

17. Eastwood GM, Reade MC, Peck L, et al. Critical care nurses' opinion and self-reported practice of oxygen therapy: a survey. Aust Crit Care 2012;25:23-30.

18. Matziou V, Vlahioti E, Perdikaris P, et al. Physician and nurs- ing perceptions concerning interprofessional communication and collaboration. J Interprof Care 2014;28:526-33.

19. Hussain SF, Gill M, Mahmood NB, Brown SA. Differences in attitudes and knowledge of doctors and nurses towards oxygen prescribing in a district general hospital. Proceedings of European Respiratory Society International Congress, 2017, Milan, Italy.

20. Schjorring OL, Toft-Petersen AP, Kusk KH, et al. Intensive care doctors' preferences for arterial oxygen tension levels in mechanically ventilated patients. Acta Anaesthesiol Scand 2018;62:1443-51.

21. Cousins JL, Wark PA, McDonald VM. Acute oxygen therapy: a review of prescribing and delivery practices. Int J Chron Obstruct Pulmon Dis 2016;11:1067-75.

22. Huijgen QC, Effing TW, Hancock KL, Schermer TR, Crockett AJ. Knowledge of pulse oximetry among general practicioners in South Australia. Prim Care Respir J 2011;20:456-8.

23. Ranieri VM, Rubenfeld GD, Thompson BT, et al. Acute respiratory distress syndrome: the Berlin definition. JAMA 2012;307:2526-33.

24. Ringbaek TJ, Terkelsen J, Lange P. Outcomes of acute exacerbations in COPD in relation to pre-hospital oxygen therapy. Eur Clin Respir J 2015;2.

25. de Alencar Neto JN. Morphine, oxygen, nitrates, and mortality reducing pharmacological treatment of acute coronary syndrome: An evidence-based review. Cureus 2018;10:2114.

26. Siemieniuk RAC, Chu DK, Kim LH, et al. Oxygen therapy for acutely ill medical patients: a clinical practice guideline. BMJ 2018;363:4169.

27. Panwar R, Hardie M, Bellomo R, et al. Conservative versus liberal oxygentation targets for mechanically ventilated patients. A pilot multicenter randomized controlled trial. Am J Respir Crit Care Med 2016;193:43-51.

28. Stokes Y, Vandyk A, Squires J, et al. Using Facebook and LinkedIn to recruit nurses for an online survey. West J Nurs Res 2019;41:96-110.

29. Shaver LG, Khawer A, Yi Y, et al. Using Facebook advertising to recruit representative samples: Feasibility asessment of a cross-sectional survey. J Med Internet Res 2019;21:14021. 